\title{
Nanomole per Liter per Microgram per Meter Squared
}

National Cancer Institute

\section{Source}

National Cancer Institute. Nanomole per Liter per Microgram per Meter Squared. NCI

Thesaurus. Code C119465.

A unit of concentration equal to nanomoles per liter, divided by micrograms per meter squared. 